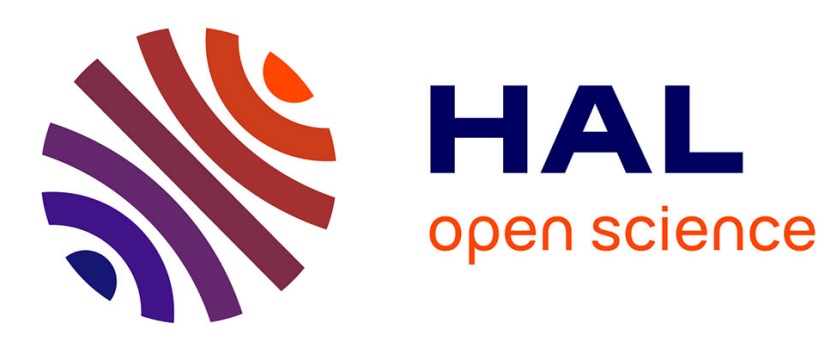

\title{
2D Traveling Wave Driven Piezoelectric Plate Robot for Planar Motion
}

Hassan Hariri, Yves Bernard, Adel Razek

\section{To cite this version:}

Hassan Hariri, Yves Bernard, Adel Razek. 2D Traveling Wave Driven Piezoelectric Plate Robot for Planar Motion. IEEE/ASME Transactions on Mechatronics, 2018, 23 (1), pp.242 - 251. 10.1109/TMECH.2018.2791508 . hal-01705415

\section{HAL Id: hal-01705415 \\ https://hal.science/hal-01705415}

Submitted on 9 Feb 2018

HAL is a multi-disciplinary open access archive for the deposit and dissemination of scientific research documents, whether they are published or not. The documents may come from teaching and research institutions in France or abroad, or from public or private research centers.
L'archive ouverte pluridisciplinaire HAL, est destinée au dépôt et à la diffusion de documents scientifiques de niveau recherche, publiés ou non, émanant des établissements d'enseignement et de recherche français ou étrangers, des laboratoires publics ou privés. 


\title{
2D Traveling Wave Driven Piezoelectric Plate Robot for Planar Motion
}

\author{
Hassan Hariri, Yves Bernard, and Adel Razek, Fellow, IEEE
}

\begin{abstract}
In this paper, a concept design of a novel Traveling Wave Driven Piezoelectric Plate Robot (TWDPPR) for planar motion is presented. The TWDPPR consists of an aluminium plate structure, with four non-collocated piezoelectric patches bonded on its surface. A two dimensions modeling of noncollocated piezoelectric patches bonded on thin structures developed and validated in previous work is used in this paper to model the TWDPPR based on the "two modes excitation" method for propulsion. A preliminary design is presented and the model is then used to verify the generation of the $2 \mathrm{D}$ traveling wave on the plate for planar motion. A prototype is fabricated and a forward, backward, \& steering motions are experimentally achieved. An experimental characterization of the TWDPPR is given in this paper.
\end{abstract}

Index Terms-Piezoelectric robot, $2 \mathrm{D}$ traveling wave, finite element modeling, two modes excitation

\section{INTRODUCTION}

$\mathbf{P}$ IEZOELECTRIC miniature motors are widely presented in literature [1]-[15]. Some of them comprise a fixed part on which are fixed the piezoelectric transducers and a movable part (slider) placed in contact with the fixed part, so as to be moved by the vibration of the piezoelectric transducers. These piezoelectric motors are not adapted for moving the fixed part on which are fixed the piezoelectric transducers, in particular because the piezoelectric transducers are not suitable to generate a wave in the whole of the fixed part. Other piezoelectric miniature motors (Linear ultrasonic motors) can only move in one direction and do not allow to move objects in a plane. The proposed piezoelectric miniature robot in this article is inspired by the linear ultrasonic motors with the difference is that there is no slider, the vibrator itself moves as an entire robot and in multi-directions. Our proposed robot is within the scope of robots that move on a solid substrate (smooth and flat ground) where many designs and mechanisms can be found in literature [16]-[25]. We are interested in the mechanism that uses the propagation of waves to generate motion and then uses the waves to control the direction of motion. Two types of waves are used for propulsion of piezoelectric miniature robots on a solid substrate, the standing wave and the traveling wave. A standing wave bi-directional linearly moving miniature piezoelectric robot is presented in [26]-[28]. Ferreira et al in [29], [30] presents a multi-degree of freedom standing wave miniature piezoelectric robot. A traveling wave miniature piezoelectric robot for bi-directional motion is presented by Hariri et al in [31]-[33]. Jones et al in

Authors are with the GeePs Group of electrical engineering-Paris, UMR CNRS 8507, CentraleSuplec, Univ. Paris-Sud, Universit Paris-Saclay, Sorbonne Universits, UPMC Univ Paris 06, 3 \& 11 rue Joliot-Curie, Plateau de Moulon 91192 Gif-sur-Yvette CEDEX, France e-mail: haririhas@gmail.com. Manuscript received April 19, 2005; revised August 26, 2015.
[34] presents a multi-degree of freedom traveling wave miniature piezoelectric robot for an underwater propulsion. Koza et al in [35] suggests a traveling wave Flagellar swimming using piezoelectric bimorph actuators. Abadi et al in [36] presents a multi-degree of freedom traveling wave miniature piezoelectric robot to control the location of the electrode in deep brain stimulation. A "flying" carpet near a horizontal surface based on piezoelectric traveling wave generation is presented by Jafferis et al in [37].

When a standing wave is used for propulsion, the legs are needed in order to transfer the up and down wave vibration into a linear motion. However, the legs are not necessarily needed when a traveling wave is used for propulsion of piezoelectric miniature robots. In the case of standing wave propulsion, special locations for the legs should be selected to prevent motion against propulsion direction of the robot. Also, legs shapes, dimensions and mechanical properties affect the experimental behaviour of the piezoelectric miniature robot where they need to be taken into account in the model for precise design which complicate the model. Miniature piezoelectric robots that use standing waves for propulsion are generally faster than the ones that use traveling waves and can move on a rougher surface because the legs increase the displacements and therefore the locomotion step of the miniature piezoelectric robots. Standing wave is easily generated in a finite length structure, however traveling wave is generated by superposition of two standing waves with $90^{\circ}$ phase shift in time and space which makes it more complicated to generate compared to a standing wave. Besides from the mentioned advantages and disadvantages of each type of propulsion (standing wave or traveling wave) relative to each other, a traveling wave miniature piezoelectric robot is more suitable for miniaturization than a standing wave one as the legs are not used. For the above mentioned reason, a traveling wave miniature piezoelectric robot is opted to study in this paper.

All the above mentioned traveling wave miniature piezoelectric robots and others cited in [31]-[40] are using 1D traveling wave on a beam structure for propulsion. However in this paper, a $2 \mathrm{D}$ traveling wave on a plate structure is used for propulsion. A $2 \mathrm{D}$ traveling wave generated on a plate structure is recently reported in [41] for future drag reduction manipulation where two piezoelectric Macro-fiber composite actuators are used to experimentally study the generation of the $2 \mathrm{D}$ traveling wave on the plate. In [41], the 2D traveling wave generated will be used in the future to control the interaction between the fluid and the plate structure to improve the efficiency of the systems studied and not to propel the whole system. There is no evidence in this work if this 2D traveling wave can be used as a driven mechanism to propel the 
entire system. The aim in this paper is to generate a mechanical traveling wave in a finite flexible plate structure to propel the plate in different directions using piezoelectric patches. The objective here is not to optimize the generation of the traveling wave on the plate but to provide a prove of concept that a 2D traveling wave generated on a finite plate structure can lead to propel the plate in different directions. Our idea is to generate a traveling wave in a plate structure to move it on a solid substrate (smooth and flat ground) using piezoelectric patches bonded on the same face of the plate surface. The proposed design consists of an elastic thin plate structure, with four non-collocated piezoelectric patches bonded on its surface as shown in Fig.1. This system is called herein by Traveling

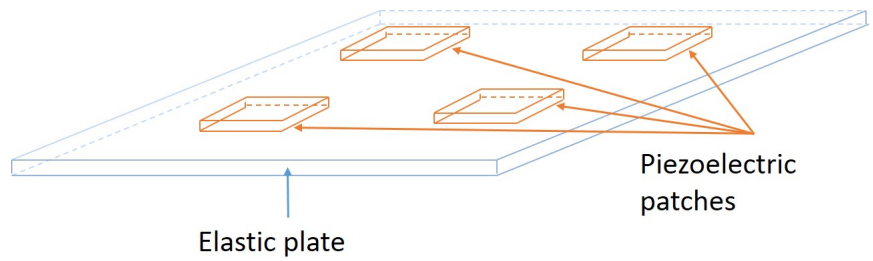

Fig. 1: A schematic for the TWDPPR

Wave Driven Piezoelectric Plate Robot (TWDPPR) for planar motion. The traveling wave on the plate is generated using the "two modes excitation" method defined in [32] and it is redefined in this paper.

After introducing the operation principle for the TWDPPR for planar motion, modeling of the TWDPPR will be briefly presented using the "two modes excitation" method based on previous finite element model. A preliminary design is shown thereafter to prove that a mechanical traveling wave can be generated on a plate and propel it in different directions. Then, the generation of the 2D traveling wave on the TWDPPR is verified by simulation using the finite element model of the TWDPPR. Based on the preliminary design results, a prototype of the TWDPPR is fabricated to verify experimentally the propulsion in different directions on smooth flat surface. The TWDPPR is experimentally characterized in terms of speed versus applied voltages, speed versus payloads, blocking forces at different applied voltage, and steering capability in different directions versus applied voltages and payloads.

\section{Operational PRinciple of the TWDPPR}

Pure traveling waves are usually observed on long structures and rarely on normal finite structures. In finite structures, the excited vibration wave is partially reflected when it hits the boundaries, which creates a mixture of standing and traveling waves. Two approaches can be found in the literature to excite traveling wave in finite structures using two piezoelectric transducers. The first approach is used in [32], [42]-[44] where one piezoelectric transducer is used to generate the traveling wave on a beam and a second piezoelectric transducer is used to prevent the reflection of the wave by means of a passive electrical circuit. The passive electrical circuit used in this approach can be replaced by active control methods like feedback control or adaptive control to control the vibrating wave along the beam and it is used in [37], [45], [46]. The second approach is an easy way to generate a traveling wave in finite structure and it belongs to the active control methods. It consists in generating a traveling wave on the structure by one piezoelectric transducer and removes the reflected wave at its boundaries by applying forces using the other piezoelectric transducer. This second approach is used in [32], [47]-[49] where both piezoelectric transducers are used to excite simultaneously two neighboring natural mode shapes of the beam at the same frequency, but with a phase difference of $90^{\circ}$. The vibration can be approximated as the superposition of these two modes; this is called a "two modes excitation". This principle is necessary to generate the traveling wave on a beam structure, and it was first demonstrated theoretically and verified also experimentally by Loh et al. in [47]. At the resonance frequency, two progressive waves with the same amplitude propagating in opposite directions cancel each other, resulting in a standing wave on the beam. This is being experimentally verified and theoretically demonstrated by Kim et al. in [49].

The operation principle for the 2D Traveling Wave Driven Piezoelectric Plate Robot (TWDPPR) for planar motion is based on the second approach and is called "two modes excitation" method. The piezoelectric patches are used in this case to excite the plate using the "two modes excitation" method at a frequency between two resonance frequencies in order to generate a traveling wave on the plate. The traveling wave is the origin of the frictional forces exerting at the contact lines between the plate and the ground that led to movement of the TWDPPR in different directions. In order to understand this concept, the case of a thin beam structure is taken (Fig.2) where the coordinate system is shown in Fig.2a. According to the Euler-Bernoulli hypothesis of thin beam, the

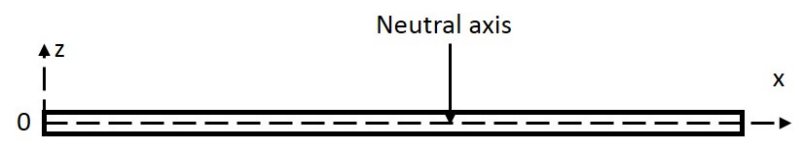

(a) The coordinate system of a thin beam

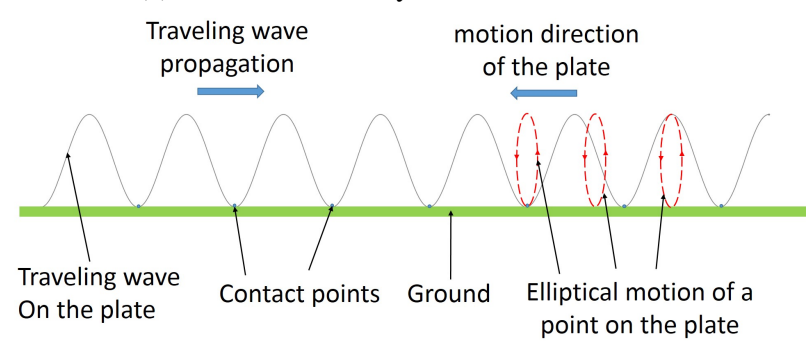

(b) Contact mechanics between the plate and the ground

Fig. 2: Traveling wave description on a thin plate structure

$x-z$ coordinate system is established by taking the neutral axis at the middle of the beam (zero point) where no extension or contraction in the $x$ direction is assumed. A pure traveling wave on the beam is defined by

$$
u_{z}=A \cos (k x-w t)
$$

where $u_{z}$ is the displacement field in the $z$ direction, $A$ is the amplitude, $k$ is the wavenumber and $\mathrm{w}$ is the angular 
frequency of the exciting signal. Therefore, according to the Euler-Bernoulli theory the displacement field in the $x$ direction $u_{x}$ can be expressed as

$$
u_{x}=-z \frac{\partial u_{z}}{\partial x}=z A k \sin (k x-w t) .
$$

Therefore

$$
\left(\frac{u_{x}}{z A k}\right)^{2}+\left(\frac{u_{z}}{A}\right)^{2}=1
$$

Evidently, the motion of a point on the beam in the $x-z$ plane is an ellipse, which is the definition of a 1D traveling wave. Fig. $2 \mathrm{~b}$ illustrates the contact mechanics between the plate and the ground. The elliptical motion of each point is the origin of the driving frictional forces between the plate and the ground that led to movement of the TWDPPR. Here we suppose that the driving friction forces are produced by the highest points of the elliptical motions as illustrated in Fig. $2 b$ and detailed discussions about that can be found in [50]. The contact points shown in Fig. $2 b$ become contact lines when the width of the plate is taken into account. Therefore, the motion of a line on the plate is an elliptic cylinder, which is the definition of a 2D traveling wave. The elliptical motions of these contact lines between the plate and the ground are that led to movement of the TWDPPR. The number of the contact lines is equal to half the number of the antinodes for a given operating frequency. Fig. 3 represents the methods of operation of the TWDPPR where a forward motion (Fig. 3a), a forward steering (Fig. 3b $\&$ Fig. 3c) and a clockwise rotational motion (Fig. 3d) are shown. The arrows in Fig. 3 indicate the motion direction of the TWDPPR.

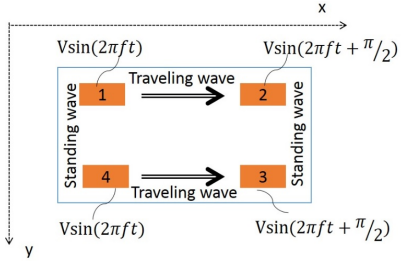

(a) Forward motion

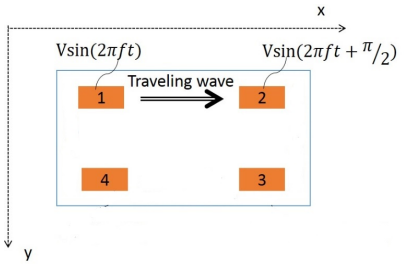

(c) Forward-Down motion

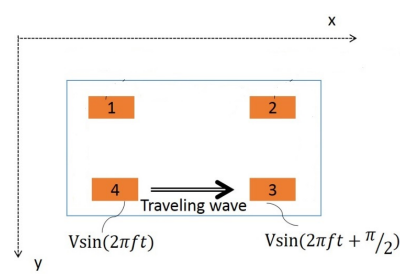

(b) Forward-Up motion

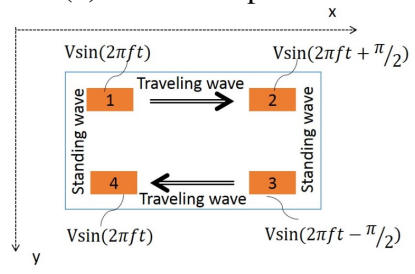

(d) Clockwise rotational motion
Fig. 3: The methods of operation of the TWDPPR

A sinusoidal signal of amplitude $V$ and frequency $f$ is applied to the piezoelectric patches with a phase difference. A phase difference of $90^{\circ}$ between patches will generate a traveling wave, and a phase difference of $0^{\circ}$ or $180^{\circ}$ between patches will generate a standing wave as shown in Fig. 3. The traveling waves are used to move the TWDPPR and the standing waves have the effect of limiting the friction between the TWDPPR and the ground on which the TWDPPR moves. To reverse moving the TWDPPR, simply reverse the phase shift or the active/inactive piezoelectric patches as indicated by Fig. 3. Different plate structures and piezoelectric patches configurations can be found in this patent [51].

\section{MODELING OF THE TWDPPR}

A finite element model for non-collocated piezoelectric patches bonded on thin structures developed and validated in [52] is retaken here and adopted to demonstrate the generation of the traveling wave on the TWDPPR using the "two modes excitation" method.

Given the geometry of the plate where the thickness is very small compared to the other two dimensions, and with the assumptions of very small deformations (less than a fifth of the thickness) and low bi-polar electric field applied to the piezoelectric patches (less than $+-0.4 \mathrm{kV} / \mathrm{mm}$ ), this finite element model is based on Kirchhoff-Love hypothesis, linear constitutive relations, plane stress formulation and Hamilton principle to obtain the variational equation governing the mechanical and piezoelectric part of the system. Then Hermite elements are used to obtain the variational equation in matrix form, taking into account the boundary conditions and the damping behaviour of the real system. In the case of "two modes excitation" method, all piezoelectric patches $\left(p_{1}, p_{2}, p_{3}, p_{4}\right)$ are used as actuators where four sinusoidal voltages are applied with the same amplitude $V$ and frequency $f$ and different phase $\phi\left(V \sin \left(2 \pi f t+\phi_{1}\right), V \sin \left(2 \pi f t+\phi_{2}\right), V \sin (2 \pi f t+\right.$ $\left.\left.\phi_{3}\right), V \sin \left(2 \pi f t+\phi_{4}\right)\right)$ while no external loads are applied. The dynamic equation is given in Eq. 4 where $M_{m m}, C_{m m}$ and $K_{m m}$ are the structural mass matrix, the structural damping matrix and the structural stiffness matrix respectively, $K_{m v p 1}, K_{m v p 2}$, $K_{m v p 3}$ and $K_{m v p 4}$ are the piezoelectric stiffness matrices for piezoelectric patches $p_{1}, p_{2}, p_{3}$ and $p_{4}, K_{v m p 1}, K_{v m p 2}, K_{v m p 3}$ and $K_{v m p 4}$ are the transpose of $K_{m v p 1}, K_{m v p 2}, K_{m v p 3}$ and $K_{m v p 4}$, and $K_{v v p 1}, K_{v v p 2}, K_{v v p 3}$ and $K_{v v p 4}$ are the dielectric stiffness for piezoelectric patches $p_{1}, p_{2}, p_{3}$ and $p_{4}$. The mass and stiffness matrices included all the geometric parameters and physical properties of the system where readers can refer to [52] for more details. $U_{i}$ is the vector with nodal structural displacements, $Q_{p 1}, Q_{p 2}, Q_{p 3}$ and $Q_{p 4}$ are the electrical charges for piezoelectric patches $p_{1}, p_{2}, p_{3}$ and $p_{4}, E_{3 p 1}, E_{3 p 2}$, $E_{3 p 3}$ and $E_{3 p 4}$ are the electric fields for piezoelectric patches $p_{1}, p_{2}, p_{3}$ and $p_{4}, V$ is the applied voltage, $t_{p}$ is the thickness of the piezoelectric patches, $f$ is the applied frequency and $\phi_{1}$, $\phi_{2}, \phi_{3}$ and $\phi_{4}$ are the phase of the applied sinusoidal voltages 
for $p_{1}, p_{2}, p_{3}$ and $p_{4}$.

$$
\begin{aligned}
& {\left[\begin{array}{ccccc}
M_{m m} & 0 & 0 & 0 & 0 \\
0 & 0 & 0 & 0 & 0 \\
0 & 0 & 0 & 0 & 0 \\
0 & 0 & 0 & 0 & 0 \\
0 & 0 & 0 & 0 & 0
\end{array}\right]\left[\begin{array}{c}
\ddot{U}_{i} \\
t_{p} \ddot{Q}_{p 1} \\
t_{p} \ddot{Q}_{p 2} \\
t_{p} \ddot{Q}_{p 3} \\
t_{p} \ddot{Q}_{p 4}
\end{array}\right]+\left[\begin{array}{ccccc}
C_{m m} & 0 & 0 & 0 & 0 \\
0 & 0 & 0 & 0 & 0 \\
0 & 0 & 0 & 0 & 0 \\
0 & 0 & 0 & 0 & 0 \\
0 & 0 & 0 & 0 & 0
\end{array}\right]} \\
& \times\left[\begin{array}{c}
\dot{U}_{i} \\
t_{p} \dot{Q}_{p 1} \\
t_{p} \dot{Q}_{p 2} \\
t_{p} \dot{Q}_{p 3} \\
t_{p} \dot{Q}_{p 4}
\end{array}\right]+\left[\begin{array}{ll}
K_{m m}-\sum_{a=1}^{4} K_{m v p a} K_{v v p a}^{-1} K_{v m p a} & K_{m v p 1} K_{v v p 1}^{-1} \\
-K_{v v p 1}^{-1} K_{v m p 1} & K_{v v p 1}^{-1} \\
-K_{v v p 2}^{-1} K_{v m p 2} & 0 \\
-K_{v v p 3}^{-1} K_{v m p 3} & 0 \\
-K_{v v p 4}^{-1} K_{v m p 4} & 0
\end{array}\right. \\
& \left.\begin{array}{rrr}
K_{m v p 2} K_{v v p 2}^{-1} & K_{m v p 3} K_{v v p 3}^{-1} & K_{m v p 4} K_{v v p 4}^{-1} \\
0 & 0 & 0 \\
K_{v v p 2}^{-1} & 0 & 0 \\
0 & K_{v v p 3}^{-1} & 0 \\
0 & 0 & K_{v v p 4}^{-1}
\end{array}\right]\left[\begin{array}{c}
U_{i} \\
t_{p} Q_{p 1} \\
t_{p} Q_{p 2} \\
t_{p} Q_{p 3} \\
t_{p} Q_{p 4}
\end{array}\right] \\
& =\left[\begin{array}{c}
0 \\
E_{3 p 1}(t)=\frac{V}{t_{p}} \sin \left(2 \pi f t+\phi_{1}\right) \\
E_{3 p 2}(t)=\frac{V}{t_{p}} \sin \left(2 \pi f t+\phi_{2}\right) \\
E_{3 p 3}(t)=\frac{V}{t_{p}} \sin \left(2 \pi f t+\phi_{3}\right) \\
E_{3 p 4}(t)=\frac{V}{t_{p}} \sin \left(2 \pi f t+\phi_{4}\right)
\end{array}\right] .
\end{aligned}
$$

As example,when $\phi_{1}=\phi_{4}=0^{\circ}$ and $\phi_{2}=\phi_{3}=90^{\circ}$ a traveling wave in the negative $\mathrm{x}$-direction and a standing wave in the negative $y$-direction are generated. Therefore, a forward motion should be achieved in the positive $\mathrm{x}$-direction for the TWDPPR. By changing $\phi_{2}$ and $\phi_{3}$ from $90^{\circ}$ to $-90^{\circ}$ a backward motion in the negative $\mathrm{x}$-direction must achieved.

The dynamic finite element model for the TWDPPR presented in this section is taken in free space, without environmental interactions. Modeling the interaction between the TWDPPR and the ground (contact mechanics) using Coulomb friction model, viscous friction model or viscoelastic foundation model is omitted at this stage to concentrate more on the design of the TWDPPR (section IV), on the generation of a $2 \mathrm{D}$ traveling wave on the plate (section $\mathrm{V}$ ), and on the experimental verification of the traveling wave on the TWDPPR (section VI). Also, as we have not developed mathematical models of the contact mechanics, the operational characteristics of the TWDPPR is obtained experimentally (see section VII).

\section{DESIGN OF THE TWDPPR}

The design process consists of determining the optimum geometry and mechanical properties for the TWDPPR that lead to optimize the generation of the traveling wave on it. It consists of determining the optimal shape and geometry of the plate and the piezoelectric patches, the material used for the plate and the piezoelectric patches, and the locations of the piezoelectric patches. Only, a preliminary design is shown in this section to prove that a mechanical traveling wave can be generated on a plate and propel it in different directions. Therefore, the aim here is not to optimize the generation of the traveling wave on the plate, but to provide a prove of concept that a $2 \mathrm{D}$ traveling wave can be generated on a plate and would lead to propel the plate in different directions.

At this preliminary stage, the shape of the plate is taken rectangular having a length of $180 \mathrm{~mm}$, a width of $60 \mathrm{~mm}$ and a thickness of $1 \mathrm{~mm}$. The type of material used for the plate is studied in [52] where a comparison is made between steel, aluminium, brass and acrylic. By using the same methodology as in [53], it was found that at $0.5 \mathrm{~mm}$ plate thickness, the aluminium is the best elastic material that have the best compromise between maximum transverse displacement (deflection in z-direction) and maximum blocking force. Therefore, the aluminium is selected for the plate.

Piezoelectric ceramic Lead zirconate titanate (PZT) is chosen here because of its marked piezoelectric effect. Among PZTs, the NCE41 from Noliac inc. is chosen because it is characterized by low self heating at high frequency operation. The NCE41 is a hard-doped PZT with low dielectric and mechanical losses and considerably high curie temperature $\left(284{ }^{\circ} \mathrm{C}\right)$. The polarization of the $P Z T$ and the applied electric field on it are chosen in the thickness direction which allow the $d_{31}$ transversal mode of vibration of the $P Z T$. The transversal mode of vibration of a PZT bonded on a flexible plate structure causes the bending of the plate. The length and width of the $P Z T$ are not optimized at this preliminary stage. The length of the PZT is chosen to be, approximately, twice its width. The length of the $P Z T$ is equal to $32 \mathrm{~mm}$ and the width is equal to $17 \mathrm{~mm}$. The thickness of the $P Z T$ is critical to the displacement of the plate and it needs to be optimized because for very thin $P Z T$, no displacements are generated on the TWDPPR while when the thickness becomes large, the bending stiffness of the TWDPPR becomes more important than the bending moment generated by the PZT patches [54]. The thickness of the PZT depends on the thickness of the plate and the material used for it. For $0.5 \mathrm{~mm}$ aluminium thickness, it was found in [52] that the maximum transverse displacement is obtained at $0.45 \mathrm{~mm}$ thickness of PZT. A $0.45 \mathrm{~mm}$ thickness for the PZT is chosen.

The effect of the positions of $P Z T$ patches on the performance of the traveling wave is studied in [33] in the case of a beam structure. It has been found that locating the PZT patches near the ends of the beam structure will lead to best performance and that the traveling wave is mainly generated on the distance between the patches. The difference in traveling wave performance for different positions of the PZT patches was due to the establishment of the $P Z T$ patches relative to the nodes and the anti-nodes of a given frequency. The locations of the piezoelectric patches are not optimized at this preliminary stage. They are chosen similar to the case of a beam structure in the $\mathrm{x}$-direction based in previous results. The locations in the $\mathrm{x}$-direction are $x_{p 1}=x_{p 4}=24 \mathrm{~mm}$ and $x_{p 2}=x_{p 3}=124 \mathrm{~mm}$ where $x_{p 1}, x_{p 2}$, $x_{p 3}$ and $x_{p 4}$ are the positions in the $\mathrm{x}$-axis of $P Z T$ patches $p_{1}$, $p_{2}, p_{3}$ and $p_{4}$ respectively. The positions of the PZT patches in the y-direction are arbitrary chosen near the ends where $y_{p 1}=y_{p 2}=3.5 \mathrm{~mm}$ and $y_{p 3}=y_{p 4}=39.5 \mathrm{~mm}$. Table I resumes the mechanical properties of the TWDPPR and its geometry. According to the maximum peak to peak electric field beyond which the PZT patch is depolarized $(+-0.3 \mathrm{kV} / \mathrm{mm}$ from Table I), a maximum applied voltage of $135 \mathrm{~V}$ amplitude 
TABLE I: Properties and geometry of the TWDPPR

\begin{tabular}{|c||c|c|}
\hline Properties, geometry & $P Z T(p)$ & Aluminium $(\mathrm{m})$ \\
\hline Young's modulus $(\mathrm{Pa})$ & - & $69 \times 10^{9}$ \\
Poisson's ratio & - & $v_{m}=0.33$ \\
Volume density $\left(\mathrm{kg}^{-3} \mathrm{~m}^{-3}\right)$ & $\rho_{p}=7900$ & $\rho_{m}=2700$ \\
Relative permittivity & $\varepsilon_{33 r}=1282$ & - \\
Frequency constant $\left(\mathrm{m} \cdot \mathrm{s}^{-1}\right)$ & $\mathrm{N}_{t}=2000$ & - \\
Piezoelectric constant $\left(\mathrm{m} \cdot \mathrm{V}^{-1}\right)$ & $d_{31}=-1.3 \times 10^{-10}$ & - \\
Elastic compliances $\left(\mathrm{Pa}^{-1}\right)$ & $\mathrm{E}_{\operatorname{maxpp}}=600$ & - \\
Max peak to peak electric field $\left(\mathrm{V} \cdot \mathrm{mm}^{-1}\right)$ & $\sigma_{11}=1.3 \times 10^{-11}, S_{12}=-4.76 \times 10^{-12}$ & - \\
Max compressive strength $(\mathrm{Pa})$ & $32 \times 17 \times 0.45$ & - \\
Length $\times$ width $\times$ thickness $\left(\mathrm{mm}^{3}\right)$ & $24,124,124,24$ & - \\
$x_{p 1}, x_{p 2}, x_{p 3}, x_{p 4}(\mathrm{~mm})$ & $3.5,3.5,39.5,39.5$ & - \\
$y_{p 1}, y_{p 2}, y_{p 3}, y_{p 4}(\mathrm{~mm})$ & & \\
\hline
\end{tabular}

(bi-polar $+-135 \mathrm{~V}$ ) must be applied to protect the PZT patch from depolarization. At this applied voltage (bi-polar $+-135 \mathrm{~V})$, the non-linear behavior and hysteresis of the PZTs patches can be neglected under bi-polar and low frequency (below the frequency constant in Table I) operations according to the manufacturers of PZTs. This is a worthy idea to verify in further research. A maximum bi-polar voltage of $+-60 \mathrm{~V}$ $(+-0.13 \mathrm{kV} / \mathrm{mm})$ was chosen in simulations and experiments as a caution.

\section{VERIFICATION OF THE 2D TRAVELING WAVE ON THE TWDPPR}

To verify the $2 \mathrm{D}$ travelling wave on the TWDPPR, we begin by determining the resonance frequencies of the TWDPPR. Using the model represented in equation (4), the resonance frequencies and mode shapes of the TWDPPR are given by

$$
\left(\left[K_{m m}\right]-\left(2 \pi f_{n}\right)^{2}\left[M_{m m}\right]\right)\left\{U_{i}\right\}=0
$$

By exciting the piezoelectric patches at the optimal operating frequency of the TWDPPR with phase difference similar to Fig. 3 presented in section II, a linear, rotational and steering motions are expected. In order to determine the optimal operating frequency, iterative simulations are performed at each frequency between two successive resonance frequencies. This frequency is taken in simulation as the middle of the two successive resonance frequencies. The excitation frequencies shown in Table II are determined using Eq.(5) and taken here as examples to justify the choice of the optimal operating frequency of the TWDPPR. For verification purpose, the six different frequencies given in Table II are excited with phase difference similar to Fig. 3a. Therefore, a 2D traveling wave is expected along the surface of the plate and standing waves are expected at both width ends of the plate.

TABLE II: The excitation frequencies of the TWDPPR

\begin{tabular}{|c||c|c|c|}
\hline Mode order $(\mathrm{n})$ & $f_{n}(\mathrm{~Hz})$ & $f_{n+1}(\mathrm{~Hz})$ & Excitation freq. $(\mathrm{Hz})$ \\
\hline 5 & 567.47 & 593.15 & 580.31 \\
20 & 2298 & 2371.9 & 2334.95 \\
42 & 5059.2 & 5219.2 & 5139.2 \\
90 & 11392 & 11420 & 11406 \\
123 & 15641 & 15845 & 15743 \\
155 & 20304 & 20372 & 20338 \\
\hline
\end{tabular}

One length end and one width end of the plate are taken to visualize the traveling wave and standing wave on the TWDPPR resulting from the simulation. Fig. 4 represents the transverse displacement in $\mu m$ through the length and width of the plate in $\mathrm{mm}$ at each instant. (Fig. 4a \& Fig. 4b), (Fig. 4c \& Fig. 4d), (Fig. 4e \& Fig. 4f), (Fig. 4g \& Fig. 4h), (Fig. 4i $\&$ Fig. 4j) and (Fig. 4k \& Fig. 4l) show the expected traveling wave at one length end of the plate and the expected standing wave at one width end of the plate at $580.31 \mathrm{~Hz}, 2334.95 \mathrm{~Hz}$, $5139.2 \mathrm{~Hz}, 11406 \mathrm{~Hz}, 15743 \mathrm{~Hz}$ and $20338 \mathrm{~Hz}$ respectively.

As mentioned in section II, a mixture between standing and traveling waves is expected on the TWDPPR because pure traveling waves are usually observed on long structures and rarely on normal finite structures. By comparing Fig. 4a, Fig. 4c, Fig. 4e, Fig. 4g, Fig. 4i and Fig. 4k with a pure traveling wave, it is noted that the traveling wave performance is increased with the excitation frequency for the length end of the plate where a good traveling wave is found at $11406 \mathrm{~Hz}$ and above and a wave closest to the standing wave is found at $580.31 \mathrm{~Hz}$ and $2334.95 \mathrm{~Hz}$. By comparing Fig. 4b, Fig. 4d, Fig. 4f, Fig. 4h, Fig. 4j and Fig. 41 with a pure standing wave, it is noted that a good standing wave performance is demonstrated for the width end of the plate at all the excitation frequencies, although it is slightly decreased when increasing the frequency. These results verify the generation of the traveling waves at the length ends of the plate and the standing waves at the width ends of the plate. They can partially verify the operation principle of TWDPPR presented in section II and shown in Fig. 3. In addition, from Fig. 4, the operating frequency of the TWDPPR is expected to be between mode order 90 \& $91(11406 \mathrm{~Hz}$ from Table II), mode order 91 \& 92, and so on. Having a good traveling wave performance with less displacement does not lead to move the TWDPPR. Also, having a good displacement with less traveling wave performance does not lead to move it as well. Good traveling wave and high transverse displacement are needed to move the TWDPPR. Therefore, the optimal operating frequency of the TWDPPR is expected to be between mode order $90 \& 91$ $(11406 \mathrm{~Hz}$ from Table II).

In order to fully verify the generation of 2D traveling wave on the TWDPPR, a top view representation in the $(x, y)$ plane for the normalized transverse displacement of the TWDPPR is shown in Fig. 5 and Fig. 6 at different time step at $20338 \mathrm{~Hz}$. The lines in the width-direction indicated the generation of a $2 \mathrm{D}$ traveling wave on the surface of the plate and the discontinuity of these lines along the length of the plate represents the rotation propulsion. The lines are not identically reproduced because a pure traveling wave is difficult to obtain in finite structure (plate) and only a mixture between standing and traveling wave is obtained. From figures, it can be observed that the traveling wave is mainly concentrated between the piezoelectric patches in the longitudinal direction. Fig. 5 shows the forward motion of the TWDPPR where a backward traveling wave is generated on the surface of the plate as indicated by the circles shown on the figure. Fig. 6 shows the clockwise rotation of the TWDPPR where an anticlockwise wave is generated on the plate as indicated by the circles shown on the figure. 


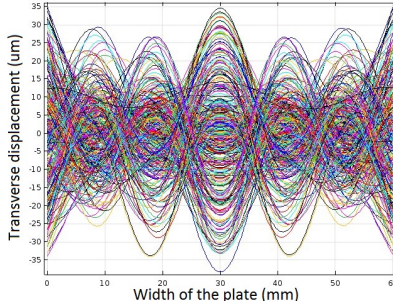

(a) $580.31 \mathrm{~Hz}$

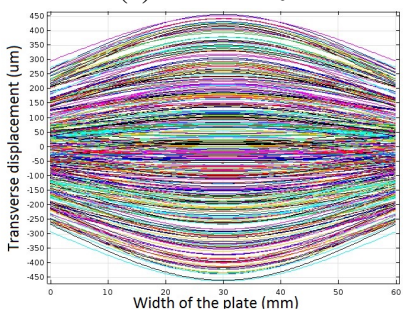

(c) $2334.95 \mathrm{~Hz}$

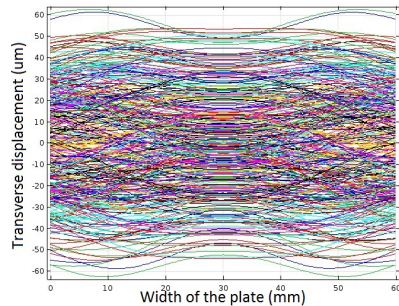

(e) $5139.2 \mathrm{~Hz}$

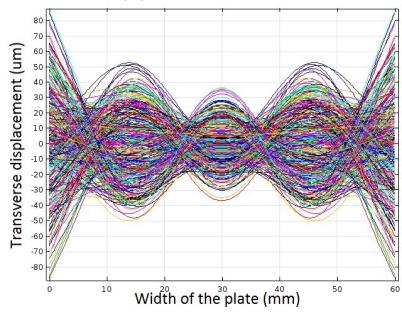

(g) $11406 \mathrm{~Hz}$

(i) $15743 \mathrm{~Hz}$

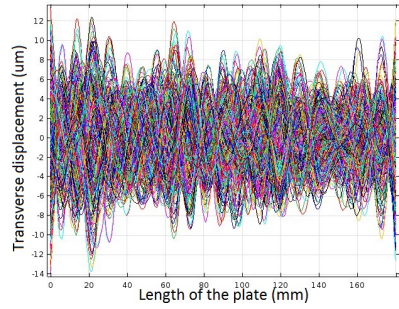

(k) $20338 \mathrm{~Hz}$

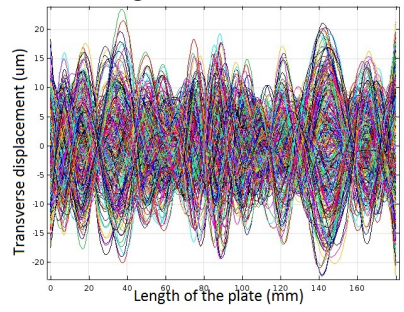

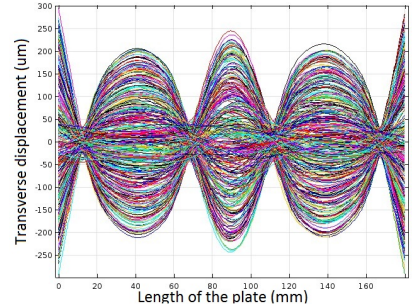

(b) $580.31 \mathrm{~Hz}$

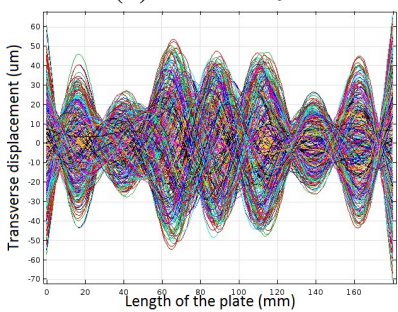

(d) $2334.95 \mathrm{~Hz}$

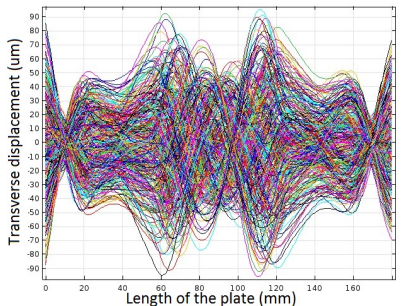

(f) $5139.2 \mathrm{~Hz}$

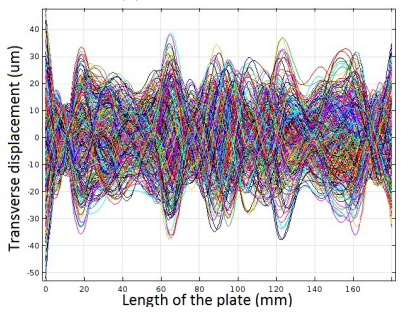

(h) $11406 \mathrm{~Hz}$

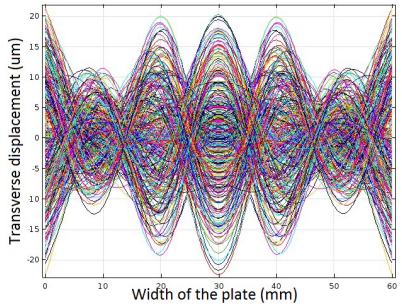

(j) $15743 \mathrm{~Hz}$

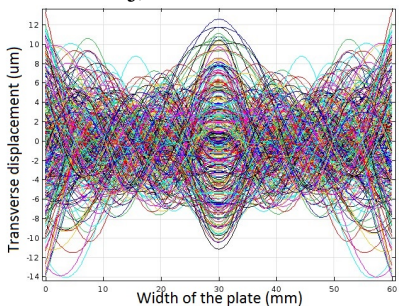

(1) $20338 H z$
Fig. 4: The expected traveling wave and standing wave on the TWDPPR at one length end and one width end for different excitation frequencies

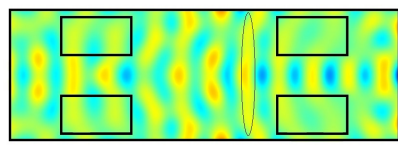

(a) $t_{1}=4896.4 \mu \mathrm{s}$

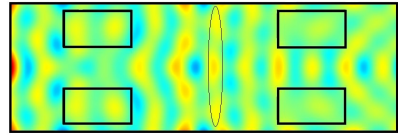

(c) $t_{3}=4904.6 \mu \mathrm{s}$

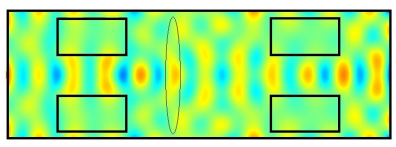

(e) $t_{5}=4912.8 \mu \mathrm{s}$

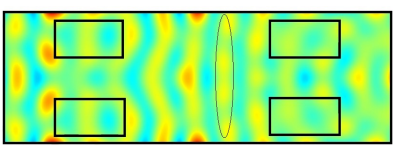

(b) $t_{2}=4900.5 \mu \mathrm{s}$

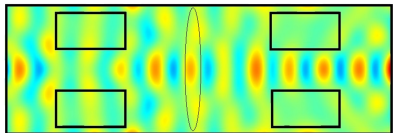

(d) $t_{4}=4908.7 \mu \mathrm{s}$

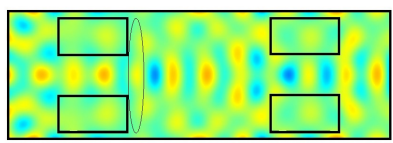

(f) $t_{6}=4916.9 \mu \mathrm{s}$
Fig. 5: Top view representation of the forward motion in the $(x, y)$ plane for the normalized transverse displacement of the TWDPPR at different time step at $20338 \mathrm{~Hz}$

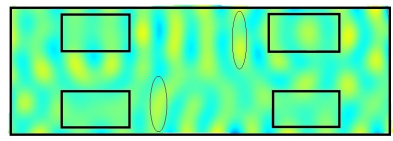

(a) $t_{1}=4896.4 \mu \mathrm{s}$

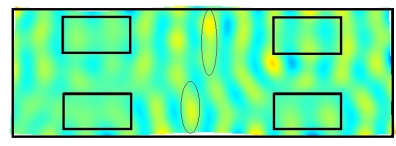

(c) $t_{3}=4904.6 \mu \mathrm{s}$

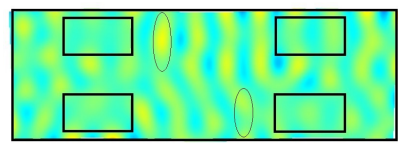

(e) $t_{5}=4912.8 \mu \mathrm{s}$

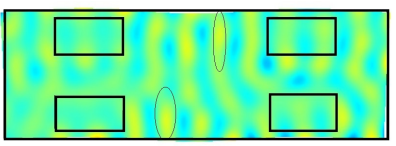

(b) $t_{2}=4900.5 \mu \mathrm{s}$

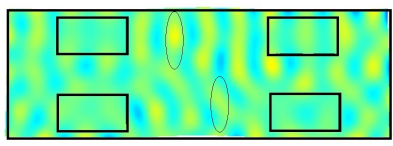

(d) $t_{4}=4908.7 \mu \mathrm{s}$

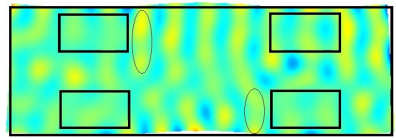

(f) $t_{6}=4916.9 \mu \mathrm{s}$
Fig. 6: Top view representation of the clockwise rotational motion in the $(x, y)$ plane for the normalized transverse displacement of the TWDPPR at different time step at $20338 \mathrm{~Hz}$

\section{FABRICATION AND EXPERIMENTAL VERIFICATION OF THE TWDPPR}

Based on the chosen geometry and mechanical properties from Table I, four PZT patches are bonded onto the aluminium plate using a strong bonding epoxy adhesive (two parts Araldite 2013 provided by Huntsman International LLC). The PZT patches are Wrapped Around Electrodes type. This type has both electrodes on the same face. Then, thin wires are soldering on the $P Z T$ patches electrodes. The experimental setup for the TWDPPR is shown in Fig.7 where the TWDPPR is connected to a signal generator via an electronic system. In addition to the power supply, the electronic system supplying the TWDPPR is composed of four power amplifiers, two inverters and one phase shifter. To choose the power amplifier, the maximum applied voltage to the piezoelectric patches and the maximum consumed current by the piezoelectric patches must be determined. The maximum applied voltage is chosen equal to $135 \mathrm{~V}$ (bi-polar $+-135 \mathrm{~V}$ ) according to the 


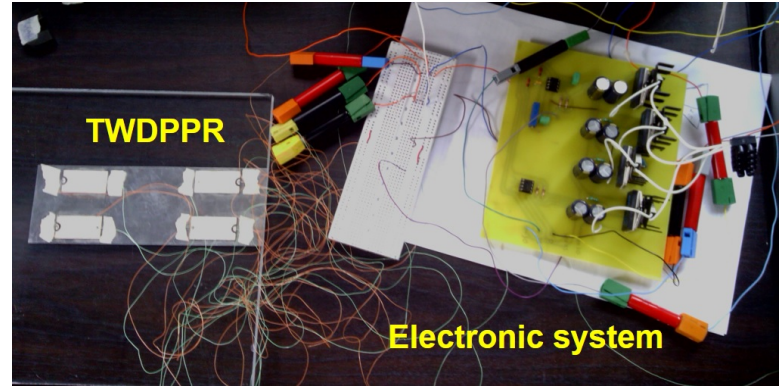

Fig. 7: An experimental setup of the TWDPPR

maximum peak to peak electric field $(+-300 \mathrm{~V} / \mathrm{mm})$ and the thickness of the piezoelectric patches $(0.45 \mathrm{~mm})$ shown in Table I. This maximum applied voltage is taken for a design purpose only and will not be applied in experiments. The maximum consumed current by the piezoelectric patches depends on the electrical properties of the piezoelectric patches, the maximum excitation frequency and the maximum applied voltage. A maximum excitation frequency of $35 \mathrm{kHz}$ is assumed to excite high frequencies (extreme case for a design purpose only). This excitation frequency is below the frequency constants of the piezoelectric patches shown in Table I, then the piezoelectric patches can be modeled by a single capacitor measured experimentally to be $14.4 n F$. This gives a maximum consumed current equal to $430 \mathrm{~mA}$. In practice, the output current of the power amplifier should be taken at least 10 times the load current to avoid the destruction of the sinusoidal signal applied to the piezoelectric patch. The chosen power amplifier $P A 93$ from APEX precision power Inc. is verifying these conditions. A heat sink is added to the power amplifier for longterm operation. A phase shifter is designed to work between $1 \& 35 \mathrm{kHz}$. The circuit is formed by a capacitor, resistances and an operational amplifier ( $T L 082$ from Texas Instruments Inc.). The phase is shifted by varying a resistance between two ends corresponding to the operating area (between $1 \& 35 \mathrm{kHz}$ ).

On the basis of this developed non-integrated electronic system, the weight of the onboard electronics must not exceed 75 $\mathrm{g}$ (11.4g for the power amplifier, $15 \mathrm{~g}$ for all other components $\& 14 \mathrm{~g}$ for the battery). Heat sinks used on the non-integrated electronic system for long-term operation could be removed from the onboard electronics where the aluminium plate can serve as a natural heat sink for power amplifiers. The ability of the robot to carry on this weight for moderate applied voltage is demonstrated in the following section.

The setup is first configured for forward motion as shown in Fig. 3a and the excitation frequency is tuned between mode order 90 \& 91 and above. The optimal operating frequency is determined experimentally by the excitation frequency that gives the maximum speed. The maximum speed of the TWDPPR is obtained at $11.2 \mathrm{kHz}$ which is close to the excitation frequency obtained by simulation $(11406 \mathrm{~Hz}$ ). At this frequency, the 2D traveling wave on the TWDPPR has a good traveling wave with high transverse displacement as seen in Fig. 4g from the simulation results. Then, the two other configurations presented in Fig.3b and Fig.3d for steering and rotational motion are tested at the optimal operating frequency
$(11.2 \mathrm{kHz})$. The TWDPPR is tested on a smooth glass flat surface and on a smooth wood flat surface where no significant difference in speed on both surfaces is reported. Experimentally, forward and backward motion, steering forward-up and forward-down motion, steering backward-up and backwarddown motion have been obtained. However, rotational motion has not been experimentally obtained. This is due to the low transverse displacements on the surface of the plate (Fig. 6) obtained by the propagation of two opposite traveling waves, and the low torque generated from the propagation of these two traveling waves.

In a future work, in order to obtain a rotational propulsion, the shape of the plate will be changed from rectangular to square shape which will lead to amplify the torque generated. Furthermore, the rectangular piezoelectric patches will be replaced by square piezoelectric patches to favor displacements in the y-direction, and therefore to generate traveling waves in the y-direction. More details can be found in this patent [51].

Fig. 8 shows an image sequence of the TWDPPR for linear and steering motion.

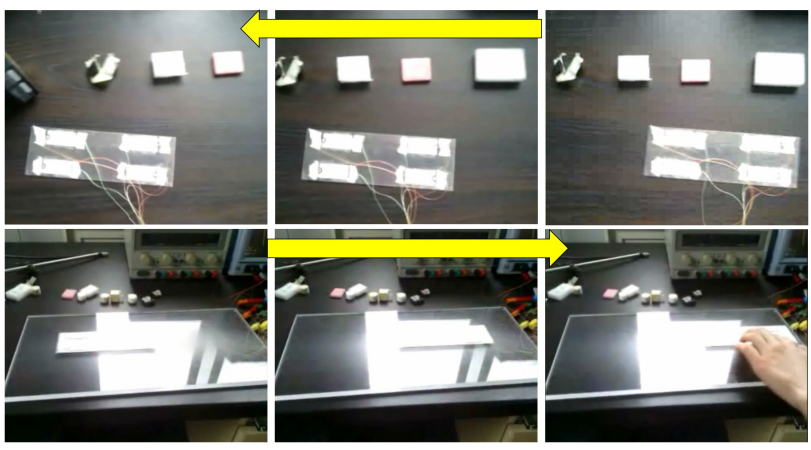

(a) Backward and forward motion

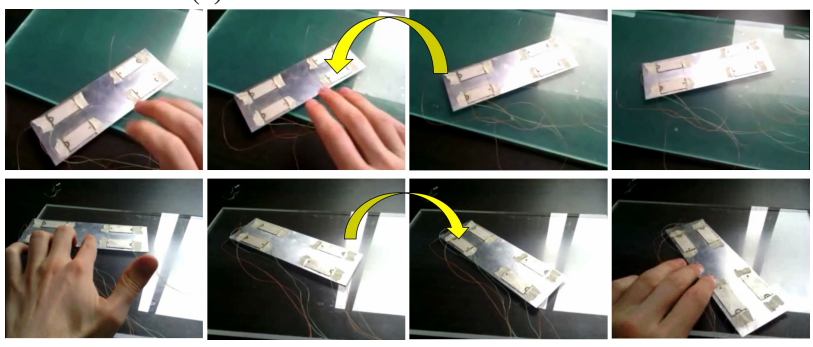

(b) Steering backward-down and forward-down motion

Fig. 8: An image sequence of the TWDPPR on smooth flat surface (wood and glass)

\section{Characterization OF THE TWDPPR}

An experimental characterization of the TWDPPR in terms of speed versus applied voltage, speed versus payload (embedded mass), blocking force and steering capability is studied in this section on a smooth glass flat surface.

A maximum applied voltage of $+-60 \mathrm{~V}$ was chosen in this experiment. Sinusoidal voltages with different phase shifts are applied to the piezoelectric patches at the optimum operating frequency $(11.2 \mathrm{kHz})$.

The average speed is computed for forward \& backward linear motions, forward-down \& backward-down steering motions. The average speed of the TWDPPR is calculated by 
the formula $d / t$ where $d$ is the distance travelled and $t$ is the time measured by a chronometer. Different voltage amplitudes are applied $(20,30,40,50,60)$ and the results are given in Fig. 9a Six trials are taken for each applied voltage, and then the average speed is calculated. Fig. 9a shows the average velocity of the TWDPPR at various applied voltages on smooth flat glass surface. It can be seen from Fig. 9a that the relationship between speed and magnitude of the applied voltage follows a linear trend. In addition, there is no clear difference in speed between forward and backward motions or forward-down and backward-down motions. This is due to the symmetry of the TWDPPR and it is in agreement with the experimental results reported in [47] for linear ultrasonic motors. The TWDPPR reaches a maximum linear speed of $133.3 \mathrm{~mm} / \mathrm{s}$ at $60 \mathrm{~V}$ amplitude. The velocity can be larger with a higher input voltage. A maximum sinusoidal applied voltage of $135 \mathrm{~V}$ amplitude can be applied without depolarizing of the piezoelectric patches.

A payload (embedded mass) is introduced onto the TWDPPR as shown Fig. 9b, and the variation of the speed versus different payloads at constant applied voltage is studied. The lightweights were located on the centre of the plate while measuring the speed. However, heavyweights were uniformly distributed on the plate while measuring the speed to balance the motion direction. The results are shown in Fig. 9c for linear forward motion and forward-down steering motion at $60 \mathrm{~V}$ amplitude. The average speed at each payload is computed in the same manner as stated above. The figure shows that the velocity decreases when the payload is increased. The slight non-linearity shown can be attributed to the payloads and their distribution on the TWDPPR affecting the operating frequency. As expected, the speed for linear forward motion is higher than for forward-down steering motion. This is because the four piezoelectric patches are excited in the first case and only two patches are excited in the second case. In order to increase the speed for steering motion, a three piezoelectric patches can be excited simultaneously [51]. The TWDPPR is able to carry $90 \mathrm{~g}$ of payload and still produce motion at $60 \mathrm{~V}$ amplitude.

The performance characteristic of the TWDPPR is essential in determining its output power,and it is specified in terms of blocking force (with velocity held to zero) and free velocity (with force held to zero), where a linear behaviour is established between them [55], [56]. The blocking force of the TWDPPR is measured experimentally on an inclined plane as shown in Fig. 9b, when the speed of the TWDPPR becomes zero. Fig. 9d shows the performance of the TWDPPR for linear forward propulsion method at different applied voltages. The nominal operating point for the TWDPPR $(17.79 \mathrm{mN}$, $66.51 \mathrm{~mm} / \mathrm{s}$ ) is determined from Fig. 9d at one half its blocking force at $60 \mathrm{~V}$ amplitude. At the nominal operating point, this TWDPPR can provide an output power of $1.18 \mathrm{~mW}$ at $60 \mathrm{~V}$ amplitude. Furthermore, the efficiency of the TWDPPR that is defined as the mechanical output power over the electrical power consumed is very high. This is because the piezoelectric patches are approximated by electrical capacitors at $11.2 \mathrm{kHz}$ (far below the frequency constant given in Table I) and therefore the electrical power consumed is considered very low. A quantitative value of the efficiency is not given herein for the lack of accurate measurement tools for measuring the phase angle between the driving voltage and the driving current that is required to compute the input power consumption in alternative operation.

Finally, the steering capability of the TWDPPR for different applied voltages and different payloads is studied. Fig. 9e and Fig. 9f show the steering motion trajectory in $(x, y)$ plane for different methods of operation (configurations) of the TWDPPR at different applied voltages and different payloads. It can be seen from figures that the steering capability of the TWDPPR is only affected by the payloads. Adding a payload into the center of mass of the TWDPPR increases the moment generated by it while moving, and thus deviates the path of movement.

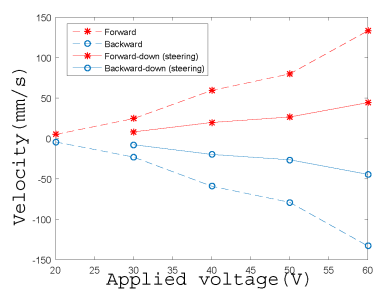

(a) Velocity vs voltage

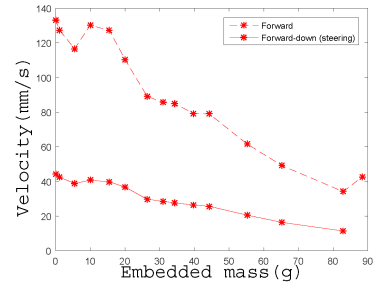

(c) Velocity vs payloads at $60 \mathrm{~V}$

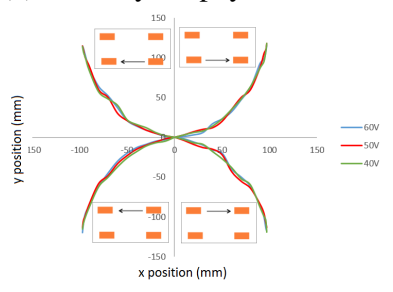

(e) Steering vs voltage

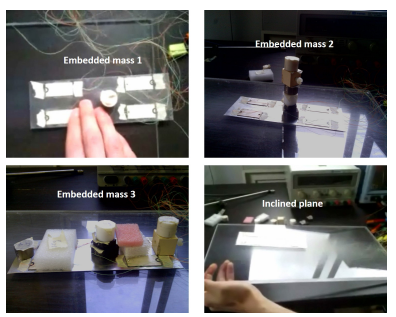

(b) With payloads \& inclined plane

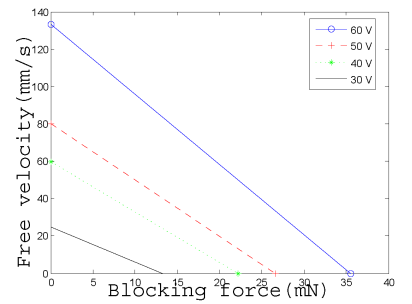

(d) Free velocity \& blocking force

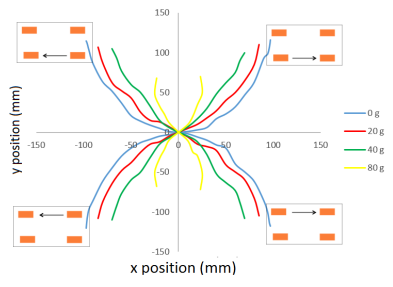

(f) Steering vs payload
Fig. 9: Characterization of the TWDPPR

With the available data, a comparison is performed against several existing Wave Driven piezoelectric miniature robots in terms of the parameters that are usually reported in the literature, such as robots dimension, mass, applied voltage, speed, output mechanical power, and planar motion capability. Table III summarizes the parameters of some comparable existing piezoelectric wave driven miniature robots. From table, miniature piezoelectric robots that use standing waves for propulsion are generally faster (bodylength/second) than the ones that use traveling waves because the legs increase the displacements and therefore the locomotion step of the miniature piezoelectric robots. However, slippage will occur during interactions between the legs and the ground in the case of standing wave driven robots and will lead to a lower 
TABLE III: Comparison to Existing Piezoelectric Wave Driven Miniature Robots.

\begin{tabular}{|c|c|c|c|c|c|c|c|}
\hline & $\begin{array}{l}\text { Dimension }(\mathbf{m m}) \\
(\mathbf{l} \times \mathbf{x} \times \mathbf{x})\end{array}$ & Mass (g) & $\begin{array}{c}\text { Peak-Peak } \\
\text { Voltage (V) }\end{array}$ & $\begin{array}{c}\text { Speed }(\mathrm{cm} / \mathrm{s}) \\
-(\text { bodylength/s) }\end{array}$ & $\begin{array}{l}\text { Output Mechanical } \\
\text { Power }(\mathrm{mW})\end{array}$ & Wave Type & Planar Motion \\
\hline Our TWDPPR Robot & $180 \times 60 \times 0.95$ & 20.4 & 120 & $13.3(0.74)$ & 1.18 & 2D Traveling wave & Yes \\
\hline LPMR [27] & $50 \times 10 \times 10.5$ & 6.27 & 200 & $30.2(6.06)$ & 0.9 & 1D Standing wave & No \\
\hline Son et al. [28] & $53 \times 45 \times 19$ & 23.25 & 20 & $5.9(1.11)$ & Not available & 1D Standing wave & Yes $^{2}$ \\
\hline Ferreira et al. [29], [30] & $64 \times 38 \times 20$ & $300^{1}$ & 56 & $30(4.69)$ & Not available & 2D Standing wave & Yes \\
\hline Hariri et al. [32] & $180 \times 17 \times 0.77$ & 6.45 & 60 & $13.1(0.73)$ & 0.43 & 1D Traveling wave & No \\
\hline
\end{tabular}

output mechanical power. It can be seen from table that a traveling wave miniature piezoelectric robot is more suitable for miniaturization than a standing wave one as the legs are not used. From the table, the robot developed in this work is the first maneuverable traveling wave driven piezoelectric miniature robot to the best of the authors knowledge.

\section{CONCLUSION}

In this paper, the design and operational principle of Traveling Wave Driven Piezoelectric Plate Robot (TWDPPR) capable of planar motion is presented. This TWDPPR consists of four piezoelectric patches bonded on a thin aluminium plate. The generation of the $2 \mathrm{D}$ traveling wave on the TWDPPR is verified using a two dimensions modeling of non-collocated piezoelectric patches bonded on thin structures developed and validated in previous work. A prototype is fabricated and an experimental verification is conducted. An experimental characterization of the TWDPPR in terms of speed versus applied voltage, speed versus payload, blocking force and steering capability is studied in this paper. The robot shows high performance in terms of maximum speed and high payloads at considerably low applied voltage which facilitates the integration of power source in future work. Further optimization of the mechanical structure to improve the $2 \mathrm{D}$ traveling wave performance will be sought in future work. This includes optimization of the piezoelectric material, in-depth investigation of the rotational motion, increasing the speed of the TWDPPR and its motion capability, as well as decrease the size and weight of the TWDPPR. Development of a skin integrated flexible electronics for the TWDPPR will also be part of our future work.

\section{REFERENCES}

[1] X. Yang, Y. Liu, W. Chen, And J. Liu, "Sandwich-Type Multi-Degree-ofFreedom Ultrasonic Motor With Hybrid Excitation", IEEE Access, Vol. 4, pp 905-913, 2016.

[2] S. C. Shen, Y. C. Chen, "Design and Evaluation of a Multi-degree-offreedom Piezoelectric Microactuator and its Applications", International Journal of Automation and Smart Technology, 2013.

[3] Z. Minghui, G. Wei, S. Lining, "A multi-degree-of-freedom ultrasonic motor using in-plane deformation of planar piezoelectric elements", Sensors and Actuators A: Physical, Vol. 148, pp 193-200, 2008.

[4] G. M Cheng, K Guo, P Zeng, and Y. M. Sun, "Development of a twodegree-of-freedom piezoelectric motor using single plate vibrator", Proc. IMechE Vol. 226 Part C: J. Mechanical Engineering Science, 2011.

\footnotetext{
${ }^{1}$ Including onboard electronics and battery.

${ }^{2}$ Two such piezo-metal composite beams are placed opposite to each other to form a pair.
}

[5] T. Ishii, R. Inoue and T. Shimizu, " $\pi$ Shaped Ultrasonic Motor with Multi-Degree of Freedom", International Journal of Control, Automation and Systems, Vol. 2, NO. 3, pp 2165-8285, 2013.

[6] Q. Pan, F. Huang, J. Chen, L. G. He, W. Li, and Z. Feng, "High-Speed Low-Friction Piezoelectric Motors Based On Centrifugal Force", IEEE Transactions on Industrial Electronics, Vol. 64, NO. 3, pp 2158-2167, 2017.

[7] Z. Xing, B. He, K. Xu, J. Wang, and S. Dong, ”A Miniature Cylindrical Piezoelectric Motor With an Asymmetric Vibrator", IEEE Transactions on Ultrasonics, Ferroelectrics, and Frequency Control, vol. 60, no. 7, pp 1498-1504, 2013.

[8] X. Li, P. Ci, G. Liu, and S. Dong, "A Two-Layer Linear Piezoelectric Micromotor", IEEE Transactions on Ultrasonics, Ferroelectrics, and Frequency Control, vol. 62, no. 3, pp 405-411, 2015.

[9] S. J. Chang and J. Chen, "Design and fabrication of the piezoelectric actuator with double pushers", Innovation, Communication and Engineering, 2014.

[10] PI, "Piezo Motor Solutions for Automation and Precision Motion Control", http://www.piezo-motor.net/, 2017.

[11] K. Kamijo, O. Miyazawa, A. Sawada, R. Nagahama, "Piezoelectric actuator, motor, robot hand, and robot", US8810109 B2 patent, 2014.

[12] K. Uchino, S. Dong, M. T. Strauss, "Piezoelectric motor and method of exciting an ultrasonic traveling wave to drive the motor", US 7095160 B2 patent, 2006.

[13] Y. Ting, Y. R. Tsai, B. K. Hou, S. C. Lin, and C. C. Lu, ”Stator Design of a New Type of Spherical Piezoelectric Motor", IEEE Transactions on Ultrasonics, Ferroelectrics, and Frequency Control, vol. 57, no. 10, 2010.

[14] S. Zhao, J. Wallaschek, "A standing wave acoustic levitation system for large planar objects", Archive of Applied Mechanics, Vol. 81, Issue 2, pp 123139, 2011.

[15] P. Ci, G. Liu, Z. Chen, and S. Dong, " A standing wave linear ultrasonic motor operating in face-diagonal-bending mode", Applied Physics Letters, 2013.

[16] D. Avirovik, B. Butenhoff and S. Priya, "Millipede-inspired locomotion through novel U-shaped piezoelectric motors", Smart Materials and Structures, 1-5, 23, 3, 2014.

[17] A. T. Baisch, "Design, Manufacturing and Locomotion Studies of Ambulatory Micro-Robots", PhD thesis, Harvard University, 2013.

[18] M. Zhou, Y. Tao, L. Cheng, W. T. Liu and X. Fu, "A Biomimetic Earthworm-Like Micro Robot Using Nut-Type Piezoelectric Motor", 6th International Conference on Intelligent Robotics and Applications, ICIRA 2013, 2013.

[19] Y. Bernard, Y. Tian and C. Hernandez, "Self moving Stick Slip piezoelectric actuator design and modelling", Proceedings of PIEZO 2011, Sestriere, Italy, 2011.

[20] H. Hariri, Y. Bernard and A. Razek, "Locomotion principles for piezoelectric miniature robots", Proceedings of ACTUATOR 10, Bremen, DE, pp. 1015-1020, 2010.

[21] T. Cimprich, F. Kaegi, W. Driesen, A. Ferreira and J. Breguet, ”Ultrasonic monolithic piezoelectric multi DOF actuators for mobile microrobots", ACTUATORO6, Bremen,DE, pp 114-117, 2006.

[22] U.Simu and S. Jhansson, "Fabrication of monolithic piezoelectric drive units for a miniature robot", Journal of Micromechanics and Microengineering, 582-589, 12, 5, 2002.

[23] H. Ishihara, T. Fukuda, K. Kosuge, F. Arai and K. Hamagishi, ”Approach to distributed micro robotic system development of micro line trace robot and autonomous micro robotic system", IEEE Int. Conf. on Robotics and Automation, ICRA95, 1995.

[24] K. Uchino, "Expansion from IT/Robotics to ecological/energy applications", ACTUATOR06, Bremen, DE, pp 48, 2006.

[25] W. Zesch, R. Bychi, A. Codourey and R. Siegwart, "Inertial drive for micro and nanorobots: two novel mechanics", Proceedings in Microrobotics 
and Micromechanical Systems, Lynne E. Parker, Editors, 80-88, 2593, 1995.

[26] H. H. Hariri, G. S. Soh, S. H. Foong, K. L. Wood and K. Otto K, "Miniature Piezoelectric Mobile Robot driven by Standing Wave", The 14th IFToMM World Congress, Taipei, Taiwan, October 25-30, 2015.

[27] H. H. Hariri, L. A. Prasetya, G. S. Soh, S. H. Foong, K. Otto and K. L. Wood, "A tether-less Legged Piezoelectric Miniature Robot using bounding gait locomotion for bidirectional motion". IEEE International Conference on Robotics and Automation (ICRA16), Stockholm, Sweden, May 16-21, 2016.

[28] K. Son, V. Kartik, J. A. Wickert and M. Sitti, "An ultrasonic standingwave-actuated nano-positioning walking robot: Piezoelectric-metal composite beam modelling", Journal of Vibration and Control, 1293-1309, $12,12,2006$

[29] A. Ferreira and J. G. Fontaine, "Dynamic Modeling and Control of a Conveyance Microrobotic System Using Active Friction Drive", IEEE/ASME TRANSACTIONS ON MECHATRONICS, 188-202, 8, 2, 2003.

[30] A. Ferreira and P. Minotti, "Control of a multidegree of freedom standing wave ultrasonic motor driven precise positioning system", Review of Scientific Instruments 68, 1997.

[31] H. Hariri, Y. Bernard and A. Razek, "Modeling and experimental study of a two modes excitation traveling wave piezoelectric miniuature robot", ACTUATOR 12, Bremen, Germany, 18-20 June, 2012.

[32] H. Hariri, Y. Bernard and A. Razek, "Traveling wave piezoelectric beam robot", Journal of Smart Materials \& structures, 1-8, 23, 2013.

[33] H. Hariri, Y. Bernard and A. Razek, "Dual piezoelectric beam robot: The effect of piezoelectric patches positions", Journal of Intelligent material systems \& structures, 1-14, 2014

[34] L. Jones, J. Spahnie, K. Lefeave, C. Haltom, A. Underwood, A. Jacob, V. V. N. S. Malladi, B. S. Joyce, P. A. Tarazaga, "Vehicle propulsion by solid state motion", Proceding of the ASME Smart Materials, Adaptive Structures and Intelligent Systems SMASIS2014, Newport, Rhode Island, USA. Sept. 8-10, 2014.

[35] G. Kosa,M. Shoham, M. Zaaroor, "Propulsion Method for Swimming Microrobots", IEEE TRANSACTIONS ON ROBOTICS, 23, 1, February, 2007.

[36] A. AbadiG. ,Kosa, "Piezoelectric Beam for Intrabody Propulsion Controlled by Embedded Sensing", IEEE/ASME TRANSACTIONS ON MECHATRONICS, 21, 3, JUNE 2016.

[37] N. T. Jafferis, J. C. Sturm, "Fundamental and Experimental Conditions for the Realization of Traveling-Wave-Induced Aerodynamic Propulsive Forces by Piezoelectrically Deformed Plastic Substrates", IEEE Journal of MicroElectroMechanical Systems, 22, 2, APRIL 2013.

[38] V. V. N. S. Malladi, D. Avirovik, S. Priya, P. A. Tarazaga, "Travelling Wave Phenomenon Through a Piezoelectric Actuation on a Free-Free Beam", Proceding of the ASME Smart Materials, Adaptive Structures and Intelligent Systems SMASIS2014, Newport, Rhode Island, USA, Sept. 8-10, 2014.

[39] V. V. N. S. Malladi, D. Avirovik, S. Priya, P. A. Tarazaga, "Characterization and representation of mechanical waves generated in piezoelectric augmented beams", Smart Materials and Structures, 24, 10, 2015.

[40] D. Avirovik, V. V. N. S. Malladi, S. Priya, P. Tarazaga, "Theoretical and experimental correlation of mechanical wave formation on beams", Journal of Intelligent Material Systems and Structures, 2016.

[41] P. F. Musgrave, V. V. N. S. Malladi, P. A. Tarazaga, "Generation of Traveling Waves in a 2D Plate for Future Drag Reduction Manipulation", In book Special Topics in Structural Dynamics, Volume 6, Part of the series Conference Proceedings of the Society for Experimental Mechanics Series, 129-138, 2016.

[42] M. Kuribayashi, S. Ueha and E. Mori, "Excitation conditions of flexural traveling waves for a reversible ultrasonic linear motor", Journal of the Acoustical Society of America, 1431-1435, 77, 4, 1985.

[43] A. Hull, "A closed form solution of a longitudinal bar with a viscous boundary condition", Journal of Sound and Vibration, 19-28, 169, 1994.

[44] V. Giurgiutu and C. Rogers, "Modal expansion modeling of the electromechanical impedance response of 1D structures", European COST F3 conference on system identification and structural health monitoring, Universidad Politecnica de Madrid, Spain, 6-9 June, 2000.

[45] S. J. Elliot and L. Billet, "Adaptive control of flexural waves propagating in a beam", Journal of Sound and Vibration, 295-310, 163, 1993.

[46] C. Mei, "The analysis and control of longitudinal vibrations from wave viewpoint", Journal of Vibration and Acoustics, 645-649, 124, 2002.

[47] B. G. Loh and P. Ro, "An object transport system using flexural ultrasonic progressive waves generated by two mode excitation", IEEE Transactions on Ultrasonics, Ferroelectrics, and Frequency Control, 994999, 47, 4, 2000.
[48] H. Jeong, G. Kim, S. B. Choi, J. H. Park, K. R. Cha, "A study of an object transport system using ultrasonic wave excitation" Journal of Mechanical Science and Technology, 941-999, 21, 2007.

[49] G. Kim, J. W. Park and S. H. Jeong, "Analysis of dynamic characteristics for vibration of flexural beam in ultrasonic transport system", Journal of Mechanical Science and Technology, 1428-1434, 23, 2009.

[50] J. Wallaschek, "Contact mechanics of piezoelectric ultrasonic motors", Smart Mater. Struct., 369-381, 7, 1998.

[51] Y. Bernard, H. Hariri, A. Razek, "Mobile piezoelectric device", Patent: WO 2015/059283, France, 2015. http://www.google.com/patents/WO2015059283A1?cl=en

[52] H. Hariri, Y. Bernard and A. Razek, "A two dimensions modeling of non-collocated piezoelectric patches bonded on thin structures", Journal of Curved and layer. Struct., 15-27, 2, 2015.

[53] M. Sitti, D. Campolo, J. Yan, R. S. Fearing, "Development of PZT and PZN-PT Based Unimorph Actuators for Micromechanical Flapping Mechanisms", 2001 ICRA IEEE International Conference on Robotics and Automation, 2001.

[54] Q. M. Wang and L. E. Cross, "Performance analysis of piezoelectric cantilever bending actuators", Ferroelectrics, 187-213, 215, 1998.

[55] A. J. Moskalik and D. Brei, "Force-deflection behavior of piezoelectric C-block actuator arrays", Smart Mater. Struct., 531-543, 8, 1999.

[56] "Introduction to piezo transdusers", Piezo Systems. www.piezo.com, 2016.

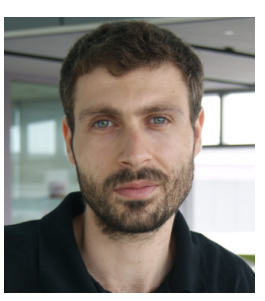

Hassan Hariri Hassan H. Hariri received the engineering degree in electrical and electronics engineering from the Lebanese University, Beirut, Lebanon, in 2009 and the Ph.D. degree from Paris-Sud University, France, in 2012. He is currently a Postdoctoral Fellow at Singapore University of Technology and Design, Singapore. Some of his many research interests include Piezoelectric devices, Biomedical devices, Miniature mobile robots, Mechanism design \& Articulated system, Biomimetic and intelligent miniature-system, Transformative reconfigurable and modular robots/Origami Robots, Digital manufacturing and 4D printing, Soft robotics, Actuators and sensors, MEMS, Smart/advanced materials, Vibration and control, Structural health monitoring, Energy harvesting, flexible electronics, robotics (including localization and navigation, vision, Artificial Intelligence, and adaptive control algorithms), and Machine-Human Interface.

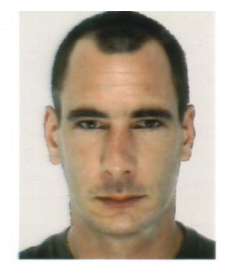

Yves Bernard Yves Bernard was born in Paris, France, on January 8, 1974. He graduated from the University of Paris-Sud Orsay with a diploma in energy transformation. He received his doctoral degree in September 2000 with a thesis on the magnetic hysteresis modeling in the finite element modeling method. He has been a professor at the University of Paris-Sud Orsay since September 2012. His research activity deals with the modeling and conception of piezoelectric devices. 


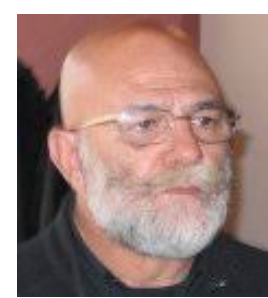

Adel Razek Adel Razek was born in Egypt. He obtained the Dipl. Eng. and M. Sc. Eng. degrees from Cairo University in Egypt in 1968 and 1971. Joining the Institut National Polytechnique de Grenoble (INPG) in 1971, he became Docteur d'Etat es Sciences Physiques in 1976. In 1977, he was a postdoctoral researcher at INPG. He has been a Research Director at the Centre National de la Recherche Scientifique (CNRS) in France since 1986. He moved to the Laboratoire de Gnie Electriquc de Paris associated with CNRS, SUPELEC, and the University of Paris, as a research scientist at CNRS in 1978, senior research scientist in 1981, research director in 1986, and senior research director in 1997. His main current research concerns computational electromagnetics (EMC, NDT, CAD) and design of electrical drives and actuators. He is the author or co-author of more than 150 scientific papers. Dr. Razek received the Andre Blondel medal in 1985 for his research work. He is a Fellow of the IEEE, a Fellow of the Institution of Electrical Engineers (IEE/IET; UK), and a Membre Emerite of the Societe des Ingenieurs Electriciens (SEE; France). 